\title{
Face Recognition-Aided IPTV Group Recommender with Consideration of Serendipity
}

\author{
Kuei-Hong Lin, Kuo-Huang Chung, Kai-Shun Lin, and Jia-Sin Chen
}

\begin{abstract}
The internet protocol television (IPTV) provide thousands of video contents to the users. However, it is difficult to each user to make selection from the amount of videos especially for the group of users. For dealing with the problem, the recommendation systems are developed to aid the users to make selection. In this study, the group recommender named "EyeTV" is proposed which is a multi-facial recognition technique-aided IPTV system, and it is an improved version of the $1^{\text {st }}$ generation proposed in 2012. The EyeTV system applies Microsoft Xbox's IP camera - KINECT to be the eye of TV for capturing facial features of users, and the multi-facial recognition technique is employed to recognize the user's identity. The users who watch the videos together are taken as a group, and then the system would memorize the watching history for that group automatically. The interactions among the group members can be predicted by the recommendation algorithm of EyeTV, that is, the recommended results are taken the members' interactions into consideration, and the recommended results may be more and more relevant to the group after training. However, the members may be able to guess what kinds of videos would be recommended to them at the time, and the group recommender can be considered as an ineffective service. For preventing the problem mentioned before and providing the group members new watching experience, the social network-based serendipity recommender is employed to improve the EyeTV system. The improved EyeTV system takes the recorded information from the social network of each member, and the data from social network would be combined with the group's watching history for providing serendipitous recommendations to the group.
\end{abstract}

Index Terms-IPTV, recommendation systems, group recommender, social network, serendipity.

\section{INTRODUCTION}

Recommender systems [1] have been developed to assist users to find items of interest from a large amount of information pool. With recommender systems, the users can browse amounts of contents efficiently and quickly. The recommendation techniques can be divided into two categories: personal recommendation [2]-[4] and group recommendation [5]-[8]. The personal recommendation has been widely used in several on-line services such as YouTube, Netflix and Amazon. The personalized recommendation service provides a convenient approach for customers to select and compare the contents and items with similar features.

Manuscript received August 30, 2013; revised November 1, 2013.

The authors are with the Information and Communications Research Laboratories of Industrial Technology Research Institute of Taiwan, R.O.C (e-mail: \{kueihong990316, khchung, itri450839, DelphiChen @itri.org.tw).
On the other hand, the group recommenders [5]-[7] can recommend to a group of users by combining information from individual user preferences and the group preferences. They consider more factors among the group members such as their interactions, opinion importance or weights in a group. Traditional group recommenders [5]-[7] have tried to generate a preference aggregation based on group members' preferences or interactions. However, the recommenders may be considered as an ineffective service finally, that is, the recommend videos can be predicted or guessed by the group members.

The EyeTV system [8] is a group recommender for recommending videos to the group members with consideration of their interactions or weights, and the rating estimates of the non-watched videos of the group would be predicted via normalized least mean square (NLMS)-based group recommender [8]. Finally, the Top- $N$ rated non-watched videos would be recommended to the group. However, the same problem would happen to EyeTV while the members' weights are tracked via NLMS algorithm [9]. For preventing this problem, the social network-based serendipity (SNS) recommender [10] is applied to improve the developed EyeTV system.

This study is organized as follows. In Section II, the components of the EyeTV system is introduced. In Section III, the proposed group recommender with consideration of serendipity would be described in detail. In Section IV, the experimental environment is built for evaluating the proposed group recommendation method. In Section V, the proposed group recommender would be compared with other recommendation methods. In Section VI, concluding comments are made.

\section{SYSTEM ARCHITECTURE}

The developed EyeTV system is shown in Fig. 1 which is composed of Microsoft Kinect internet protocol camera (IP Cam), set top box (STB) and servers: electronic program guide (EPG), metadata, video content, global user rating matrix (GURM) and proposed group recommendation algorithm.

In the client side, the open source library named OpenKinect (http://openkinect.org/wiki/Main_Page) is applied to link the STB (Intel processor: CE4100) with Kinect IP camera, and the Kinect IP camera can sense the moving objects and catch the facial features of users. The caught facial information will be sent to STB for making multi-facial recognition [11], [12] as shown in Fig. 2. Thus, the client side family members should register (Fig. 3) 
themselves for saving facial features to the family member profile (FMP). As shown in Fig. 4, the FMP of each member has an unique user identity (UID) for obtaining or writing data to the GURM which saves the global users' comments of each video content with a corresponding video identity (VID). In STB, the group profile table (GPT) and group history table (GHT) are applied to memorize UIDs in each watching group and each group's watching history, respectively. In Table I, each group has a group identity (GID), and the family members have their own weights in the group, and the weights represent the interactions among the members. The weights are expressed as the interactions among the group members [6]-[8]. For example, if group $1(\mathrm{GID}=1)$ has $\mathrm{UID}_{1}$ and $\mathrm{UID}_{2}$ as its group members, and weighting vector $\boldsymbol{W}_{1}$ is expressed as $\left[W_{1,1} W_{1,2} W_{1,3}\right]$, where the magnitude of $W_{l, 3}$ is set as 0 . Moreover, the weights of each member are estimated and updated the NLMS-based group recommender [8]. The NLMS-based group recommender [8] is the kernel design of the $1^{\text {st }}$ generation EyeTV system (proposed in 2012), and it is employed to predict the members' weights for generating the group ratings' estimates of non-watcheded videos. Note that

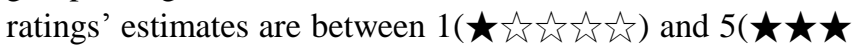
$\star \star$ ). As the other recommenders, the videos with Top- $N$ rating estimates would be recommended to the group. Therefore, the group can save their time for discussing and debating in selection the thousands of non-watched videos.

After the group rating the recommended video, GHT is updated automatically. In Table II, each group history has its rating result and the NLMS algorithm's error term of $n^{\text {th }}$ times recommendation. Note that the rating result is considered as the comment of the group to the watched video, and the NLMS's error term is memorized for generating the weighting vector in $(n+1)^{\text {th }}$ times recommendation which is calculated by subtracting the group's rating estimate from the real rating result.

In the server side, the group recommendation algorithm applies the information from the client side and other servers for making video recommendation to the group. The global user rating matrix (GURM) memorizes global uses' ratings of video contents. In Table III, the GURM saves the VID, the UID and the users' ratings of each video. Note that the

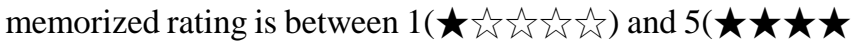
$\star)$, and the default ratings of non-watcheded videos are set as 0 . Besides, the EPG and the metadata server save the titles of contents and their extended information corresponding to the VID, respectively. In the metadata server, the data are saved as TABLE IV which is referenced the design of MovieLens (http://www.grouplens.org/node/73). Note that the extended information includes the genres of the videos, and each video may include one or more genres. Therefore, the genres are saved as the vector type

$$
\mathbf{g}(V I D)=\left[g(V I D, 1) g(V I D, 2) \cdots g\left(V I D, N_{g}\right)\right]
$$

where $\mathbf{g}(V I D)$ stands for the genre vector of the $\mathrm{VID}^{\text {th }}$ movie, $N_{g}=18$ is the number of genres, $\mathbf{g}(V I D, x)$ is the $x^{\text {th }}$ element of $\mathbf{g}(V I D)$, and the elements of the genre vector are action, adventure, animation, children's, comedy, crime, documentary, drama, fantasy, film-noir, horror, musical, mystery, romance, Sci-Fi, thriller, war and western, respectively. As shown in TABLE IV, the movie named "Toy Story" has 3 genres: animation, children's and comedy, and its

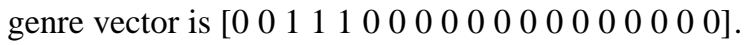

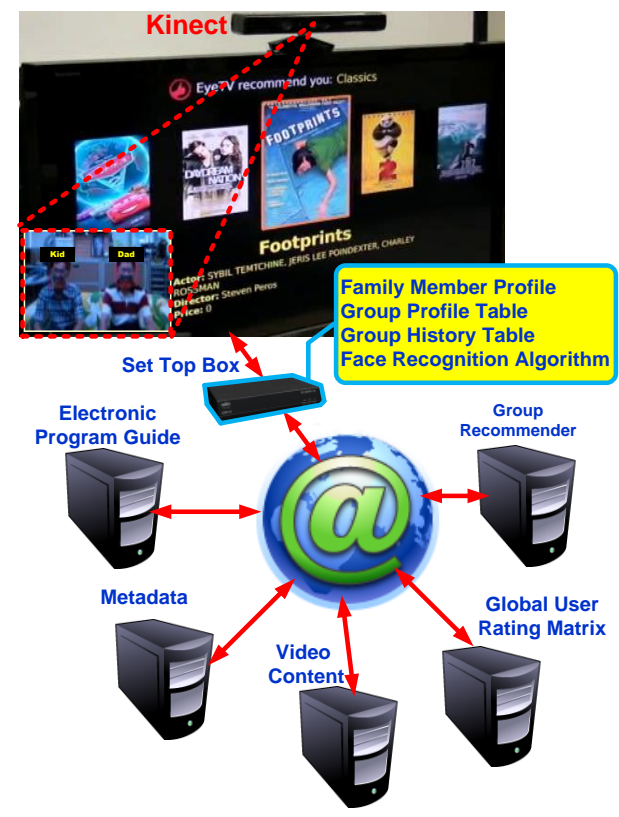

Fig. 1. EyeTV system.

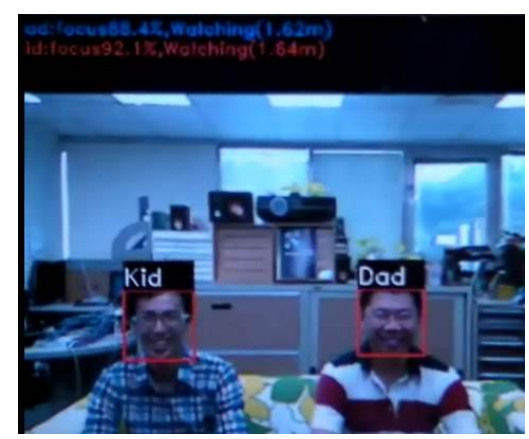

Fig. 2. Multi-facial recognition (distance between TV and users: 1.6m)

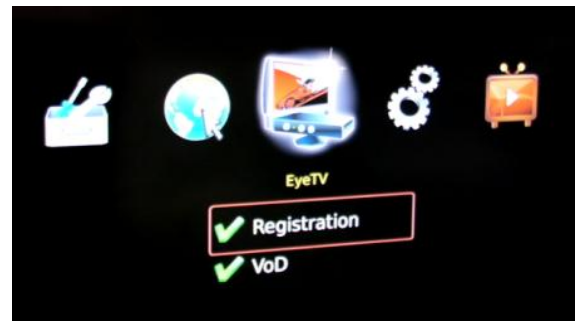

Fig. 3. EyeTV registration and video on demand functions.

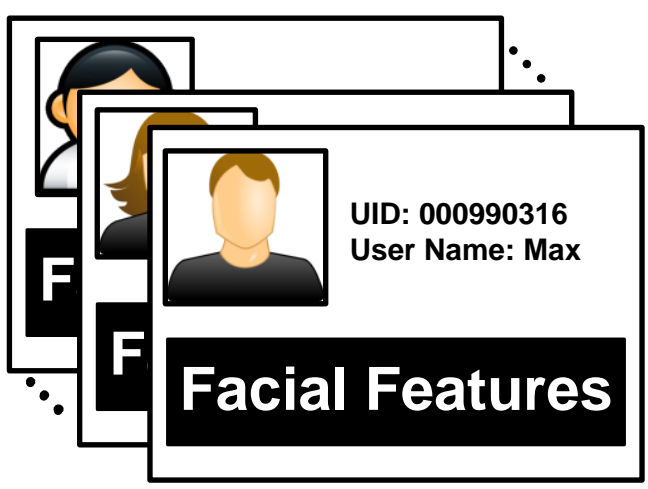

Fig. 4. Family member profile. 
TABLE I: GROUP PROFILE

\begin{tabular}{cccc}
\hline \hline $\mathrm{GID}$ & $\mathrm{UID}_{1}$ & $\mathrm{UID}_{2}$ & $\mathrm{UID}_{3}$ \\
\hline 1 & $W_{l, 1}$ & $W_{l, 2}$ & $W_{l, 3}$ \\
2 & $W_{2,1}$ & $W_{2,2}$ & $W_{2,3}$ \\
$:$ & $:$ & $:$ & $:$ \\
$N_{G I D}$ & $W_{G, 1}$ & $W_{G, 2}$ & $W_{G, 3}$ \\
\hline \hline
\end{tabular}

TABLE II: GROUP HISTORY

\begin{tabular}{|c|c|c|c|c|c|}
\hline GID & VID & $\begin{array}{l}\text { Recommendatio } \\
\mathrm{n} \text { Index }\end{array}$ & $\begin{array}{l}\text { Group's } \\
\text { Rating }\end{array}$ & $\begin{array}{c}\text { Members' } \\
\text { Rating }\end{array}$ & $\begin{array}{c}\text { NLMS } \\
\text { error } \\
\text { term: } e(n)\end{array}$ \\
\hline 1 & 10 & 1 & 2 & {$\left[\begin{array}{lll}1 & 1 & 2\end{array}\right]$} & 2 \\
\hline 1 & 22 & 2 & 3 & {$\left[\begin{array}{lll}3 & 3 & 2\end{array}\right]$} & 3 \\
\hline 2 & 19 & 1 & 5 & {$\left[\begin{array}{llll}4 & 5 & 4 & 5\end{array}\right]$} & 1 \\
\hline 2 & 202 & 2 & 3 & {$\left[\begin{array}{llll}2 & 3 & 2 & 2\end{array}\right]$} & 2 \\
\hline$:$ & $:$ & $:$ & $:$ & : & : \\
\hline$K$ & 234 & 1 & 2 & {$\left[\begin{array}{ll}1 & 2\end{array}\right]$} & 2 \\
\hline
\end{tabular}

TABLE III: GLOBAL USER RATING MATRIX

\begin{tabular}{c|cccc} 
VID & & & & \\
$\boldsymbol{U I D} \backslash$ & $\boldsymbol{1}$ & $\boldsymbol{2}$ & $\cdots$ & $\boldsymbol{N}$ \\
\hline $\boldsymbol{I}$ & $R_{1,1}$ & $R_{1,2}$ & $\cdots$ & $R_{1, N}$ \\
$\boldsymbol{2}$ & $R_{2,1}$ & $R_{2,2}$ & $\cdots$ & $R_{2, N}$ \\
$\vdots$ & $\vdots$ & $\vdots$ & $\vdots$ & $\vdots$ \\
$\boldsymbol{M}$ & $R_{M, 1}$ & $R_{M, 1}$ & $\cdots$ & $R_{M, N}$
\end{tabular}

TABLE IV: METADATA

\begin{tabular}{|c|c|c|}
\hline VID & Title & Genre Vector \\
\hline 1 & Toy Story (1995) & 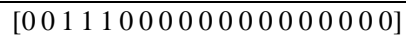 \\
\hline 2 & $\begin{array}{l}\text { Golden Eye } \\
\text { (1995) }\end{array}$ & 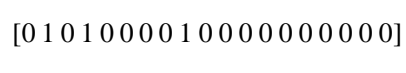 \\
\hline : & $:$ & : \\
\hline
\end{tabular}

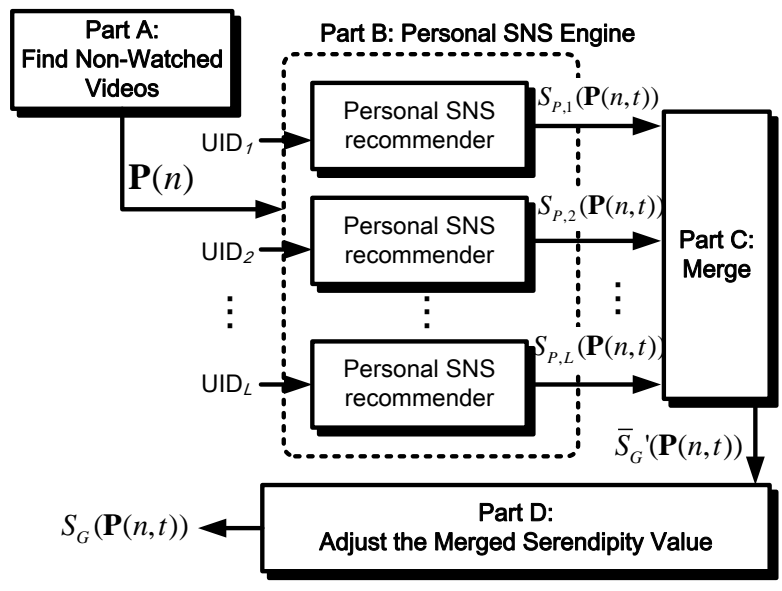

Fig. 5. Proposed group recommender with SNS engine.

\section{GROUP RECOMMENDER WITH SOCIAL NETWORK-BASED SERENDIPITY (SNS) ENGINE}

For providing the group members the serendipitous experience in IPTV group recommendation, the group recommender with social network-based serendipity (SNS) engine is proposed. In Fig. 5, the proposed SNS group recommender is divided into 4 parts for recommending the non-watched and serendipitous videos to the group.

\section{A. Part A: Find Non-Watched Videos}

For recommending non-watched videos to group members, the non-watched videos of the group members would be found by comparing the group's watched videos in GHT with EPG. The VIDs of the non-watched videos (NVIDs) expressed as

$$
\mathbf{P}(n)=\left[N V I D_{1} N V I D_{2} \cdots N V I D_{T}\right]
$$

where $n$ is the recommendation index, which is memorized in GHT, and $T$ is the number of non-watched videos.

\section{B. Part B: Personal SNS Engine}

In Fig. 5, the serendipity value (SV) of each group member corresponds to its NVID would be measured by $L$ personal SNS recommender [10], where $L$ is the number of group members.

In Fig. 6, the personal SNS recommender [10] applies the social network interaction database, access record database and social network relationship database to calculate the factors: friendship $\left(F_{p}\right)$, recency $\left(R_{y}\right)$ and frequency $\left(F_{y}\right)$ for measuring the SV of each NVID, respectively. Note that $F_{y}$ consists of 3 sub-factors: frequency of self $\left(F_{s}\right)$, friend $\left(F_{f}\right)$ and database $\left(F_{d}\right)$. Each calculated factor would be normalized, and the value is between 0 and 1 . The definition and formulation of each factor or sub-factor would be discussed as bellow:

\section{1) Friendship $\left(F_{p}\right)$}

$F_{p}$ is the interpersonal relationship. It is calculated by the interactive information between the target user and his or her friends. The definition of $F_{p}$ is formulated as

$$
\begin{aligned}
& \text { Total amount of interaction } \\
& F_{p}=\frac{\text { with target friend of target user }}{\text { Total amount of interaction }} \\
& \text { with each friend of target user }
\end{aligned}
$$

\section{2) Recency (Ry)}

$R_{y}$ is about the access time of the video, the earlier access time means more recency of item. The definition of $R_{y}$ is formulated as

$$
\begin{aligned}
\text { The access time of target item } \\
R_{y}=\frac{- \text { The earliest access time in database }}{\text { The latest access time }} \\
- \text { The earliest access time in database }
\end{aligned}
$$

\section{3) Frequency $(F y)$}

$F_{y}$ means that who access target item in self access record, or in friend's access record, or in whole database. $F_{y}$ has three sub-dimensions: frequency of self $\left(F_{s}\right)$, friend $\left(F_{f}\right)$ and database $\left(F_{d}\right)$. The calculating functions are

$$
\begin{gathered}
F_{s}=\frac{\text { The number of times target user access target item }}{\text { Total amount of access times of target user }} \\
F_{f}=\frac{\text { The number of times target user's friends access target item }}{\text { Total amount of access times of target users friends }}
\end{gathered}
$$

and

$$
F_{d}=\frac{\text { The number of times any user access target item }}{\text { Total amount of access times of whole database }}
$$




\section{4) Conversion procedure}

The calculate factors would be passed the conversion procedure for converting the influence of each factor. The conversion procedure is formulated as

$$
x^{\prime}=1-x
$$

where $x$ is the target which is needed to be converted. For example, the $F_{y}$ of watching cartoons and actions are 0.9 and 0.1 , respectively. After converting by $(8)$, the value $F_{y}$ ' of cartoons is 0.1 , and $F_{y}$ ' of actions is 0.9 . The results show that the cartoons are not serendipitous or interesting to the group anymore, and the group recommender may need to recommend the actions to the group.

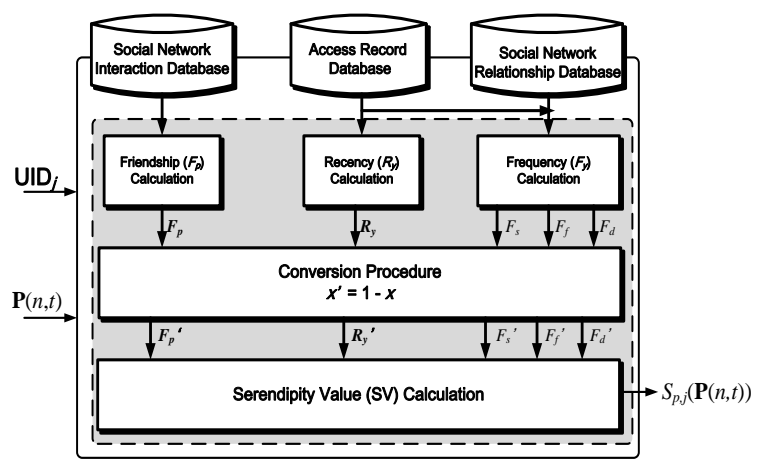

Fig. 6. Part B: personal SNS engine.

\begin{tabular}{|c|c|c|c|c|}
\hline \multirow{4}{*}{\begin{tabular}{|l} 
Social Network \\
Interaction Input \\
\\
Message-1: \\
Amy good! \\
Chris ok!
\end{tabular}} & $\begin{array}{l}\text { Social Network } \\
\text { Relation Ship Input }\end{array}$ & \multicolumn{3}{|c|}{ Access Record Input } \\
\hline & & Amy & Avatar & $1 / 1$ \\
\hline & Friends of Amv: & Bob & Avatar & $1 / 1$ \\
\hline & Bob Chris. & Chris & KungFu & $1 / 2$ \\
\hline & & Bob & Inception & $1 / 3$ \\
\hline $\begin{array}{l}\text { Message-2: } \\
\text { Amy fine! }\end{array}$ & & Chris & Inception & $1 / 3$ \\
\hline $\begin{array}{l}\text { Amy: fine! } \\
\text { Chris ok! }\end{array}$ & Friends of Bob: & Daniel & Twilight & $1 / 4$ \\
\hline & Chris & Emily & Twilight & $1 / 5$ \\
\hline Message-3: & & Daniel & Twilight & $1 / 6$ \\
\hline Amy no way! & Friends of Chris: & Emily & Twilight & $1 / 7$ \\
\hline Bob fine! & Amy, Bob & Chris & 2012 & $1 / 11$ \\
\hline Message-4: & & & & \\
\hline $\begin{array}{l}\text { Amy ok! } \\
\text { Chris good! }\end{array}$ & Friends of Daniel: & & & \\
\hline & Friends of Emily: & & & \\
\hline
\end{tabular}

Fig. 7. Example of social network interaction, relationship and access record.

\section{5) Personal serendipity value calculation}

The $t^{\text {th }}$ NVID's personal SV of the $j^{\text {th }}$ group member the is calculated by

$$
S_{P, j}(\mathbf{P}(n, t))=\left(\sum_{k=1}^{K} F_{p, k}^{\prime} / K\right) \times \alpha+\left(\sum_{m=1}^{M} R_{y, m}^{\prime} / M\right) \times \beta+\gamma F_{y}^{\prime}
$$

where the range of $S_{p, j}(\mathbf{P}(n, t))$ is between 0 and $1, \mathbf{P}(n)$ is the NVIDs (2), $K$ is the number of friends and $M$ is the number of access records of the target video in the whole database. For example, the number of friends of Amy is 2 as shown in the social network relationship of Fig. 7, and the number of access records of Inception on $3^{\text {rd }}$ January is 2 as shown in the access record of Fig.7. $F_{p}{ }^{\prime}, R_{y}{ }^{\prime}$ and $F_{y}$ ' are the conversion of $F_{p}, R_{y}$ and $F_{y}$, respectively. Note that $F_{y}$ ' consists of $F_{s}, F_{f}$ and $F_{d}$, and it can be represented as

$$
F_{y}^{\prime}=\left(\sum_{k=1}^{K} F_{s, k}^{\prime} / K\right) \times \gamma_{1}+F_{f}^{\prime} \times \gamma_{2}+F_{d}^{\prime} \times \gamma_{3}
$$

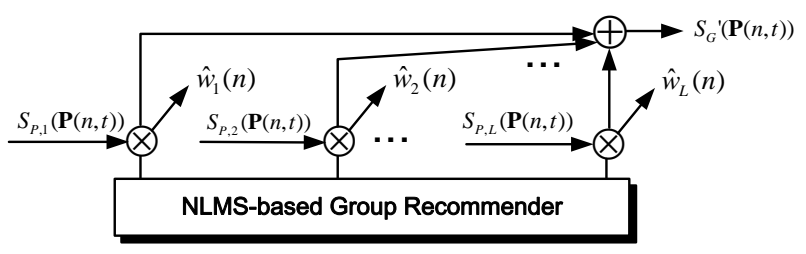

Fig. 8. Part C: merge.

where $K$ is the number of friends as shown in (9). Besides, $\alpha, \beta$, $\gamma, \gamma_{1}, \gamma_{2}$ and $\gamma_{3}$ are the weights of SV's factors in (9) and (10). The weights are set as 1/5 equally. For example, the SV of Inception in Amy's records is

$$
\begin{aligned}
\left.S_{p} \text { (Inception }\right) & =\left(\sum_{k=1}^{K} F_{p, k}^{\prime} / K\right) \times \alpha+\left(\sum_{m=1}^{M} R_{y, m}^{\prime} / M\right) \times \beta \\
& +[\underbrace{\left(\sum_{k=1}^{K} F_{s, k}^{\prime} / K\right) \times \gamma_{1}+F_{f}^{\prime} \times \gamma_{2}+F_{d}^{\prime} \times \gamma_{3}}_{\gamma F_{y}}] \\
& \left\{\begin{array}{l}
(1-0.75+1-0.25) / 2+(1-0.2+1-0.2) / 2 \\
+[(1-0.33+1-0.5) / 2+(1-0.4)+(1-0.8)]
\end{array}\right\} \\
& =\frac{0.657}{5}
\end{aligned}
$$

The $F_{p}$ with Chris for user Amy is $3 / 4=0.75$, and the $F_{p}$ with Bob for user Amy is $1 / 4=0.25$. The $R_{y}$ of Amy and Bob on $3^{\text {rd }}$ January are $(3-1) /(11-1)=0.2$ and $(3-1) /(11-1)=0.2$, respectively. The $F_{s}$ of Amy and Bob are $1 / 3$ and $1 / 2$, respectively. The $F_{f}$ is $2 / 5=0.4$. The $F_{d}$ is $2 / 10=0.2$.

\section{Part C: Merge}

As shown in Fig. 8, the $t^{\text {th }}$ NVID's personal SV (9) of each member is merged via the weighting estimates of the members, and the merged $\mathrm{SV}$ is represented as

$$
S_{G}{ }^{\prime}(\mathbf{P}(n, t))=\mathbf{S}_{P}(\mathbf{P}(n, t)) \hat{\mathbf{w}}_{N L M S}(n)
$$

where

$$
\begin{gathered}
\mathbf{S}_{P}(\mathbf{P}(n, t))=\left[S_{P, 1}(\mathbf{P}(n, t)) S_{P, 2}(\mathbf{P}(n, t)) \cdots S_{P, L}(\mathbf{P}(n, t))\right] \\
\hat{\mathbf{w}}_{\text {NLMS }}(n)=\left[\hat{w}_{1}(n) \hat{w}_{2}(n) \cdots \hat{w}_{L}(n)\right]^{T}
\end{gathered}
$$

$L$ is the number if group members. $\hat{\mathbf{w}}_{\text {NLMS }}(n)$ is the weighting vector of the members, the value of each element in $\hat{\mathbf{w}}_{N L M S}(n)(14)$ is between 0 and 1 , and $n$ is the recommendation index. The members' weights (14) are estimated by the NLMS-based group recommender [8]. The NLMS-based group recommender [8] can help the group to predict the ratings of each NVID with consideration the interactions among the members, and select the Top- $N$ videos for recommending to the group. The weighting estimates $\hat{\mathbf{w}}_{\text {NLMS }}(n)$ would be updated after the group rating to the recommended video. Thus, $\hat{\mathbf{w}}_{\text {NLMS }}(n)$ (14) can be denoted as

$$
\hat{\mathbf{w}}_{N L M S}(n)=\hat{\mathbf{w}}_{N L M S}(n-1)+\frac{\mu e^{*}(n-1) \hat{\mathbf{R}}(n-1)}{\hat{\mathbf{R}}^{H}(n-1) \hat{\mathbf{R}}(n-1)}
$$

where $n$ is the recommendation index, $\mu$ is the step size, 
$e(n-1)$ is the error term saved in GHT (TABLE II), and $\hat{\mathbf{R}}(n-1) \quad(16)$ is the memorized rating vector that corresponds to the $x^{\text {th }}$ NVID of $\mathbf{P}(n-1)$ in (2). $\quad \hat{\mathbf{R}}(n-1)$ is generated by comparing the GURM (TABLE III) via the members' UIDs or estimating by the collaborative filtering-based methods [3].

$$
\hat{\mathbf{R}}(n-1)=\left[\tilde{R}_{1, x}^{\prime}(n-1) \tilde{R}_{2, x}^{\prime}(n-1) \cdots \tilde{R}_{L, x}^{\prime}(n-1)\right]^{T}
$$

where $x$ stands for the $x^{\text {th }}$ NVID of $\mathbf{P}(n)$ (2) which is selected by the group in $(n-1)^{\text {th }}$ times recommendation. Note that the group's selected VID with its corresponding recommendation index and $\hat{\mathbf{R}}(n-1)$ are memorized in GHT's 'Members' Rating" (TABLE II) for updating the weighting vector (12) in next time, and the updated weighting vector (12) would be memorized in GPT (TABLE I).

\section{Part D: Adjust the Merged Serendipity Value}

The merged SV $S_{G}{ }^{\prime}(\mathbf{P}(n, t))$ (12) would be adjusted for preventing the group to watch the videos which compose of some genres frequently, that is, these videos with these types may not serendipitous to the group. For adjusting $S_{G}{ }^{\prime}(\mathbf{P}(n, t))$ (12), the data of GHT (TABLE II) and the metadata server (TABLE IV) are applied to make statistics. In GHT, the GID with its corresponded watched videos' VIDs are applied to generate the genre matrix by searching the corresponded VIDs' genre vector (1) in the metadata server. The genre matrix is expressed as

$$
\begin{aligned}
\mathbf{G} & =\left[\mathbf{g}\left(\mathbf{P}^{\prime}(n-1,1)\right) \mathbf{g}\left(\mathbf{P}^{\prime}(n-1,2)\right) \cdots \mathbf{g}\left(\mathbf{P}^{\prime}(n-1, n-1)\right)\right]^{T} \\
& =\left[\begin{array}{cccc}
g\left(\mathbf{P}^{\prime}(n-1,1), 1\right) & g\left(\mathbf{P}^{\prime}(n-1,1), 2\right) & \cdots & g\left(\mathbf{P}^{\prime}(n-1,1), N_{g}\right) \\
g\left(\mathbf{P}^{\prime}(n-1,2), 1\right) & g\left(\mathbf{P}^{\prime}(n-1,2), 2\right) & \cdots & g\left(\mathbf{P}^{\prime}(n-1,2), N_{g}\right) \\
\vdots & \vdots & \cdots & \vdots \\
g\left(\mathbf{P}^{\prime}(n-1, n-1), 1\right) & g\left(\mathbf{P}^{\prime}(n-1, n-1), 2\right) & \cdots & g\left(\mathbf{P}^{\prime}(n-1, n-1), N_{g}\right)
\end{array}\right]
\end{aligned}
$$

where $\mathbf{P}^{\prime}(n-1)$ is the set of the watched VIDs of the group until the (n-1) times recommendation, and it has (n-1) VIDs inside. $\mathbf{g}$ is the genre vector $(1)$, and $\mathbf{g}\left(\mathbf{P}^{\prime}(n-1, t)\right)$ stands for the genre vector of the $t^{\text {th }}$ VID in $\mathbf{P}^{\prime}(n-1)$. Note that each column of $\mathbf{G}$ (17) is one of the genre's records. For example, column 1 and 2 of $\mathbf{G}$ (17) are action's and adventure's records, respectively. The weighting vector of genres is applied to adjust the $S_{G}{ }^{\prime}(\mathbf{P}(n, t))(12)$ denoted as

$$
\mathbf{w}_{g}=\left[w_{g, 1} w_{g, 2} \cdots w_{g, N_{g}-1}\right]^{T}
$$

where $w_{g, j}=1-w_{g, j}$ ' is the $j^{\text {th }}$ element of $\boldsymbol{w}_{g}(18)$, and $\boldsymbol{w}_{g, j}$ ' is

$$
w_{g, j}^{\prime}=\frac{\sum_{i=1}^{n-1} g\left(\mathbf{P}^{\prime}(n-1, i), j\right)}{\sum_{j=1}^{N_{g}} \sum_{i=1}^{n-1} g\left(\mathbf{P}^{\prime}(n-1, i), j\right)}
$$

where $w_{g, j}$ is considered as the watching frequency of the $j^{\text {th }}$ genre, the range of $w_{g, j}$ ' is between 0 and 1 , and $g\left(\mathbf{P}^{\prime}(n-1, i), j\right)$ are the components in $\mathbf{G}$ (17). The weighting vector (18) would be applied to generate the adjusting factor for $S_{G}$, $(\mathbf{P}(n, t))(12)$. The adjusting factor is defined as

$$
\Delta(\mathbf{P}(n, t))=\mathbf{g}(\mathbf{P}(n, t)) \mathbf{w}_{g}
$$

where $\mathbf{g}(\mathbf{P}(n, t))$ is the genre vector of the $t^{\text {th }}$ VID in $\mathbf{P}(n)(2)$. The adjusted $S_{G}{ }^{\prime}(\mathbf{P}(n, t))$ is

$$
S_{G}(\mathbf{P}(n, t))=S_{G}{ }^{\prime}(\mathbf{P}(n, t)) \times \Delta(\mathbf{P}(n, t))
$$

where the range of $S_{G}(\mathbf{P}(n, t))$ is between 0 and 1 . The $S_{G}(\mathbf{P}(n$, $t)$ ) of each NVID would be collected, and a threshold (Th) would be applied to filter the NVIDs with lower $S_{G}(\mathbf{P}(n, t))$. Note that the range of $T h$ is between 0 and 1 . Finally, the serendipitous and fresh videos would be provided to the group.

\section{EXPERIMENT SETUP}

The Movie Lens (http://movielens.umn.edu/) dataset is applied to test the proposed group recommendation method. UIDs in the utilized database are from 1 to 6040, and movie IDs are from 1 to 3592 . Note that the movie IDs are corresponded to VIDs. The Movie Lens database memorize the Use rid, Moved, Rating and Timestamp ratings of each watching record, and each movie is memorized with its Movie ID, Title and Genres. Note that the Genres are saved as the genre vector (1).

In Table $\mathrm{V}$, simulation parameters for testing the proposed group recommendation method are given. The simulated members of groups are selected randomly, and the number of simulated groups is 1000. Each group member have 100 co-rated movies to be utilized in the experiments. Each group let $50 \%$ co-rated movies be the training data, and $50 \%$ co-rated movies be the testing data. The training data is applied to catch the group members' weighting estimates (15), and the testing data is applied to evaluate the proposed group recommender. The group members' weights are generated randomly each time, and $F_{p}$ of each member to their friends are random numbers between 0 and 1 , where the members' friends are selected from the database randomly.

Note that the number of friends of each member is between 10 and 200. The factors $\left(R_{y}, F_{s}, F_{f}\right.$ and $\left.F_{d}\right)$ of $\mathrm{SV}$ of each member are calculate via the provided information from the database. Note the Timestamps of Movie Lens are applied to generate the access records as shown in Fig. 7, and $R_{y}$ is calculated by these records.

The recommendation method for comparing with the proposed group recommender is random choosing (RC) [10]. $\mathrm{RC}$ is a traditional recommendation method which selects the NVIDs for providing to the group randomly, and it can be considered as providing SV to the NVIDs by random values between 0 and 1 . The recommended times $(100 \times 50 \%=50)$ of $\mathrm{RC}$ and the proposed group recommender are the same, and Th for filtering the group SVs (21) is between 0.5 and 0.6.

The Serendipity (SRDP) [8], [13] is applied to evaluate the proposed group recommender. The definition of SRDP is

$$
S R D P=\frac{\text { Useful items of unexpect recommendations }}{\text { Total unexpect recommendations }}
$$

where SRDP is used to show the efficiency of all recommendations, the larger $S R D P$ value means the method provides more number of useful items to the user. 
TABLE V: SIMULATION PARAMETERS

\begin{tabular}{lc}
\hline \multicolumn{1}{c}{ Parameters } & Settings \\
\hline Range of UID & $1 \sim 6040$ \\
Range of UID & $1 \sim 3592$ \\
Range of ratings & $1 \sim 5$ \\
Number of group members (NGM) & 2 \\
Number of friends per group member & $10 \sim 200$ \\
Threshold (SV) for filtering the & $0.5 \sim 0.6$ \\
Number of tested groups & 1000 \\
Number of co-rated movies for the & 100 \\
Ratio of the training data & $50 \%$ \\
Ratio of the tested data & $50 \%$ \\
\hline \hline
\end{tabular}

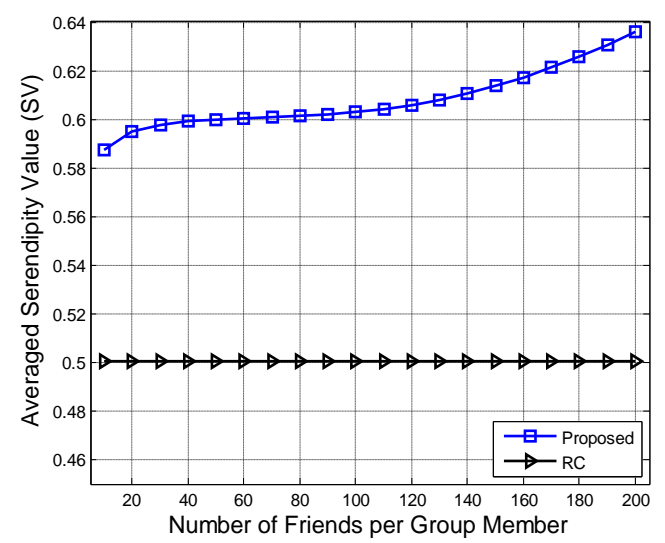

Fig. 9. Comparison of average SV under different NFGM.

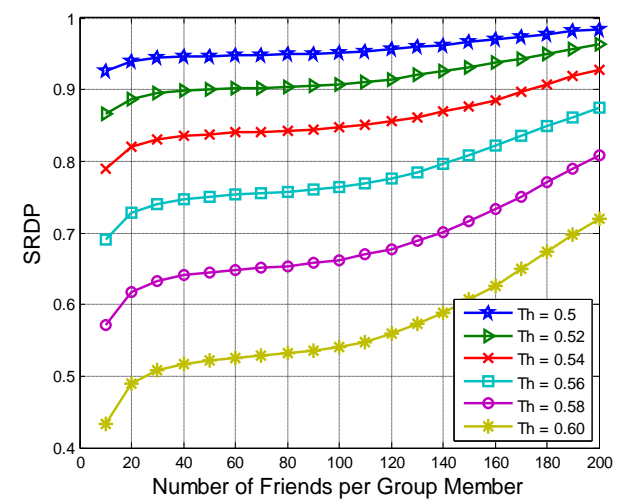

Fig. 10. SRDP under different NFGM.

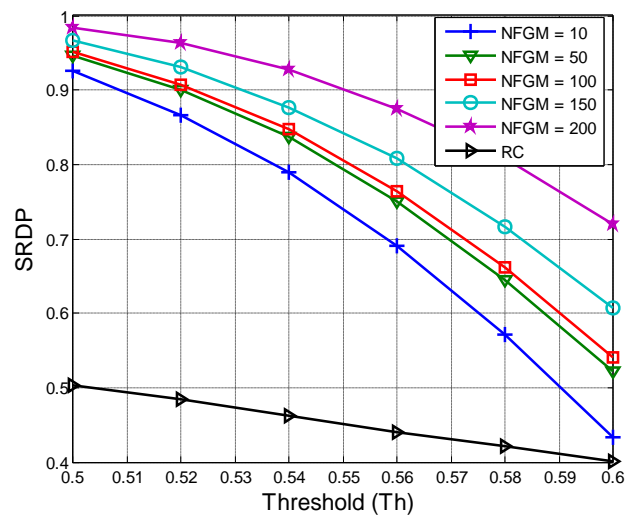

Fig. 11. SRDP under different $T h$ values.

\section{EXPERIMENT RESULTS}

In Fig. 9, the average $\mathrm{SV}$ of the proposed group recommender is higher than the RC method, that is, the proposed method has higher opportunity to get higher SRDP.

In Fig. 10, the average SV curves under different number of friends per group member (NFGM) and the different $T h$ values are shown. From the results of Fig. 10, it shows that the more NFGM can increase the SRDP effectively. Moreover, we found that the setting of $T h$ value should be lower while the number of friends per group member is lower, and it can help the groups that have little friends or some members have no social-network accounts. For example, the SRDP of NFGM $=10$ under $T h=0.6$ is lower than 0.5 , and SRDP can be increased via setting the $T h$ lower than 0.58 effectively.

In Fig. 11, the SRDP under different NFGM and different $T h$ values is compared to the RC method. With the similar results in Fig. 10, the higher NFGM can provide higher SRDP, the higher Th especially. Besides, the SRDP of the proposed group recommender is higher than the $\mathrm{RC}$ method even if it is under NFGM=10 and $T h=0.6$.

\section{CONCLUSION}

An improved EyeTV system is proposed in this study. With consideration of the serendipity, the proposed SNS-based group recommender can provide the serendipitous experience to the watching group. Unlike the traditional group recommenders, the recommendation results of the improved EyeTV system would always be fresh to the users. Thus, the improved EyeTV system provides not only the innovative IPTV service but also consideration of the user experience. Some refinements of EyeTV system would be studied continuously, and we hope the EyeTV system could be a business used IPTV service in the future.

\section{ACKNOWLEDGMENT}

This research is supported by the Information and Communications Research Laboratories (ICL), Industrial Technology Research Institute (ITRI), Taiwan, Republic of China (ITRI Grant Project Code C352BR2100).

\section{REFERENCES}

[1] F. Ricci, L. Rokach, B. Shapira, and P. B. Kantor (Eds.), Recommender Systems Handbook, New York, NY, USA, Springer, 2011.

[2] M. Balabanović and Y. Shoham, "Fab: Content-based, collaborative recommendation," Comm. ACM, vol. 40, no. 3, pp. 66-72, 1997.

[3] L. G. Terveen and W. C. Hill, "Beyond recommender systems: Helping people help each other," J. Caroll, Human-Computer Interaction, ACM Press, pp. 487-509, 2001.

[4] R. Burke, "Hybrid web recommender systems," in The adaptive web: methods and strategies of web personalization, P. Brusilovsky, A. Kobsa, W. Nejdl, Eds., Springer-Verlag, 2007, pp. 377-408.

[5] A. Jameson and B. Smyth, "Recommending to groups," in The adaptive web: methods and strategies of web personalization, $\mathrm{P}$. Brusilovsky, A. Kobsa, W. Nejdl, Eds., Heidelberg, Springer, 2007, pp. 596-627.

[6] Y. L. Chen, L. C. Cheng, and C. N. Chuang, "A group recommendation system with consideration of interactions among group members," Expert Systems with Applications: An International Journal, vol. 34, no. 3, pp. 2082-2090, April, 2008.

[7] K. H. Lin, Y. S. Chiu, and J. S. Chen, "An adaptive correlation-based group recommendation system," Intelligent Signal Processing and Communications Systems (ISPACS), no. 6146060, 2011.

[8] K. H. Lin, D. H. Shiue, Y. S. Chiu, W. H. Tsai, F. J. Jang, and J. S. Chen, "Design and implementation of face recognition-aided IPTV adaptive group recommendation system based on NLMS algorithm," in Proc. Communications and Information Technologies (ISCIT), 2012, pp. $626-631$.

[9] S. Haykin, Adaptive Filter Theory, $4^{\text {th }}$ ed., Prentice Hall, 2002, pp. 320-326. 
[10] Y. S. Chiu, K. H. Lin, and J. S. Chen, "A Social network-based serendipity recommender system," in Proc. 2011 International Symposium on Intelligent Signal Processing and Communication Systems (ISPACS), 2011.

[11] K. I. Chang, K. W. Bowyer, and P. J. Flynn, "An evaluation of multimodal 2D+3D face biometrics," IEEE Transactions on Pattern Analysis and Machine Intelligence, vol. 27, no. 4, 2005.

[12] A. Assadi and A. Behrad, "A new method for human face recognition using texture and depth information," in Proc. of 10th Symposium on Neural Network Applications in Electrical Engineering, 2010, pp. 201-205.

[13] M. Ge, C. D. Battenfeld, and D. Jannach., "Beyond accuracy: Evaluating recommender systems by coverage and serendipity," in Proc. the fourth ACM Conference on Recommender Systems, 2010, pp. 257-260.

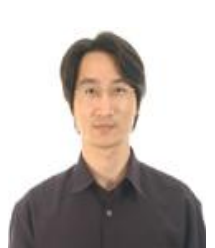

Kuo-Huang Chung received his master degree in computer science from National Sun-Yat Sen University, in 2002. He is currently pursuing the $\mathrm{PhD}$ degree at National Chiao Tung University in Computer Science. Besides, he is also a software engineer of the Industrial Technology Research Institute of Taiwan, R.O.C. His research interests include voice signal processing, video compression,

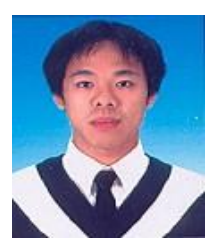

Kai-Shun Lin received the B.S. and M.D. degrees in computer science and information engineering from I-Shou University, Taiwan, in 2003 and 2005, respectively, and the $\mathrm{Ph} . \mathrm{D}$. degree in Computer Science and Information Engineering from National Chung Cheng University, Chia-Yi, Taiwan, R.O.C., in 2012. He is currently a software engineer of the Industrial Technology Research Institute of Taiwan R.O.C. His research interests include biomedical image analysis, computer vision, multimedia signal processing, and image processing.

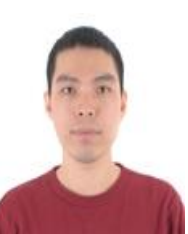

Jia-Sin Chen received his master degree in computer science and information engineering from National Taiwan University of Science and Technology in 2005. Besides, he is also a software engineer of the Industrial Technology Research Institute of Taiwan, R.O.C. His research interests include image processing, voice signal processing, and IPTV.

and IPTV. 